\title{
A New Era of Pharmacovigilance: Future Challenges and Opportunities
}

\author{
Gianluca Trifirò $^{1 *}$ and Salvatore Crisafulli ${ }^{2}$ \\ ${ }^{1}$ Department of Diagnostics and Public Health, University of Verona, Verona, Italy, ${ }^{2}$ Department of Medicine, University of Verona, \\ Verona, Italy
}

Keywords: pharmacovigilance, pharmacoepidemiology, real-world data, drug safety, regulatory science

\section{INTRODUCTION}

Medicines safety monitoring is a continuous and dynamic process throughout all the phases of the life cycle of a drug. During the drug development, safety is investigated in different phases. In preclinical studies, the primary goal of safety evaluation is the identification of a safe dose in humans and of safety parameters for clinical monitoring. In clinical phase, phase I studies are designed to estimate the tolerability of the dose range expected to be needed for later clinical studies in healthy volunteers; phase II studies are focused on determining appropriate range of drug doses in patients with a disease or condition of interest, while phase III clinical trials are the most important studies to refine understanding of benefit-risk profile of the drug and to identify less common adverse drug reactions. Although drug safety evaluation is very rigorous and thorough, pre-marketing clinical trials have however intrinsic limitations that do not allow to exhaustively evaluate drug safety profile (Singh and Loke, 2012). These studies are conducted on limited numbers of patients that are selected based on strict eligibility criteria and not fully representing real-world populations and have limited duration, thus preventing detection of rare and long-term adverse reactions.

Therefore, the post-marketing assessment of medicines plays a key role for better defining drugs' safety profile in real-world setting and filling the evidence gap of pre-marketing studies.

In the field of drug safety and regulation, a number of challenges have to be faced in the near future. First of all, COVID-19 pandemic highlighted how relevant pharmacovigilance and proper risk communication during public health emergency are. Second, the development of advanced methodologies including machine learning techniques and the availability of large amount of electronic healthcare data offer opportunity for optimizing drug benefit-risk profile evaluation in real world setting. Finally, innovative therapeutics, such as advanced therapy medicinal products, digital therapeutics, vaccines developed based on advanced technologies, requiring special pharmacovigilance monitoring have been increasingly marketed in recent years, often upon accelerated pathway approval. Some of the challenges and future opportunities in this field are briefly discussed below.

\section{PHARMACOVIGILANCE IN HEALTHCARE EMERGENCY}

Received: 31 January 2022 Accepted: 10 February 2022 Published: 25 February 2022

Citation:

Trifirò G and Crisafulli S (2022) A New Era of Pharmacovigilance: Future Challenges and Opportunities. Front. Drug. Saf. Regul. 2:866898. doi: 10.3389/fdsfr.2022.866898
During the first waves of the pandemic, the absence of vaccines and drugs for treatment/ prevention of COVID-19 led to a rush to repurpose drugs already approved for other indications. As a consequence, a large number of drugs (e.g., hydroxychloroquine, ivermectin and azithromycin) has been off-label used for the treatment of COVID-19 patients, even if underlying scientific evidence on benefits was of low quality and mostly based on in vitro studies (Sultana et al., 2020a).

Pharmacovigilance monitoring in this context has been crucial for identifying the risks associated to drugs off-label used, thus reminding the "do not harm first" principle, especially if no or weak 
evidence on benefits is available. This is the case of azithromycin, a macrolide antibiotic that has been widely used, for the treatment of COVID-19 patients (Crisafulli et al., 2021). Its known proarrhythmogenic activity, which can be exacerbated when used in combination with other drugs proposed for COVID19 treatment (e.g., hydroxychloroquine), led regulatory agencies to issue warnings against the use of this drug, unless in case of bacterial superinfection occurrence (Sultana et al., 2020b).

Accelerated approvals of drugs and vaccines to tackle the COVID-19 pandemic emphasized also the need to expeditely generate safety data in post-marketing setting by identifying and preventing serious risks and ultimately ensuring patients' safety.

Another lesson learned from COVID-19 pandemic is the importance of drug- and vaccine-related risk communication to healthcare providers as well as patients for informed therapeutic choice and ease of appropriate use of medicines/ vaccines. On the contrary, failure to effectively communicate to public and healthcare professionals can lead to a loss of trust and reputation of regulators and other stakeholders, as well as loss of lives (World Health Organization, 2020). It is the case of hydroxychloroquine, one of the drugs that gained much attention to be repurposed for COVID-19 treatment. Despite its efficacy was not proven, it has been praised by numerous public figures, such as the United States past President Donald J. Trump. As a consequence, several observational studies documented a substantial increase in purchases and internet searches for hydroxychloroquine and chloroquine after being promoted by Donald J Trump (Liu et al., 2020; Niburski and Niburski, 2020), showing how misleading information, especially if coming from individuals in positions of power, may increase inappropriate drug use and the risk of serious adverse reactions.

\section{DATABASE NETWORKS FOR POST-MARKETING SURVEILLANCE FOR VACCINES AND MEDICINES}

The increased access to large scale distributed database networks provides new ways and opportunities to monitor the postmarketing safety of vaccines and medicines and to generate real-world evidence to support decision-making. With this aim, in May 2008 the FDA launched the Sentinel Initiative, an infrastructure analyzing electronic healthcare data to assess the safety of approved medical products. To date, Sentinel has developed one of the largest distributed database networks for the assessment of medical product safety, comprising the Sentinel System, which uses common data models and analytic tools to analyze pre-existing real-world data, and the FDA-Catalyst, which uses routine queries, interventions and interactions with health plan members and/or providers (Food and Drug Administration, 2019).

Conducting pharmacoepidemiological studies combining multiple databases is particularly useful when outcomes or exposure of interest are rare, or when evidence is needed from different countries, to generate evidence rapidly and with stronger external validity (Gini et al., 2020). The combination of several claims databases may provide the statistical power needed to investigate the association between clinically relevant safety outcomes and specific drug exposure. In this regard, the Italian VALORE project is a good example of how the creation of a distributed network of administrative databases can have a great potential for conducting post-marketing surveillance of biological drugs, including biosimilars, in Italian patients affected by immunemediated inflammatory diseases (Trifirò et al., 2021).

One of the lessons learned from COVID-19 is that the potential of distributed networks of administrative databases to promptly generate robust real-world evidence is particularly high in conditions of public health emergency. This is the example of the ITA-COVID19 network, an Italian multiregional network established for the conduction of pharmacoepidemiological studies to evaluate the association between drugs, vaccines and COVID-19 through the linkage of claims databases to COVID-19 registries (Spila-Alegiani et al., 2021; Trifirò et al., 2020). Other examples of distributed networks of real-world data sources being widely used to support COVID-19 research are OpenSAFELY, an English analytics platform for analysis of electronic health records data (Williamson et al., 2020) and the Observational Health Data Sciences and Informatics (OHDSI) program, an interdisciplinary collaborative aiming at generating real-world evidence through large-scale analytics (Lane et al., 2020).

\section{ARTIFICIAL INTELLIGENCE IN PHARMACOVIGILANCE}

The availability of healthcare data has been tremendously increasing over the last years and will further increase in the near future thanks to massive marketing of digital tools collecting patient-derived data.

Huge amounts of electronic data present an opportunity to apply artificial intelligence (AI) techniques to improve drug safety assessment. Information extraction, using natural language processing (NLP) techniques and text mining to gather relevant insights from available, largely unstructured sources, has been gaining importance within the field of clinical research. As regards pharmacovigilance, text mining and NLP methods can be very useful to gather information on adverse drug reactions (ADRs) and drug-drug interactions from various textual sources, supporting researchers and clinicians in monitoring drug safety (Wong et al., 2018). Indeed, both public and private entities are currently trying to develop AI tools that can allow to automatically process ADRs (Basile et al., 2019).

Artificial intelligence and machine learning may also be useful in pharmacovigilance for 1) the automatic execution of tasks associated with case report entry and processing, 2) the identification of clusters of adverse events representing symptoms of syndromes, 3) the conduction of pharmacoepidemiological studies, 4) data linkage, through the conduction of probabilistic matching within datasets and 5) the prediction and prevention of adverse events through specific models using real-world data (Bate and Hobbiger, 2021). 


\section{SAFETY MONITORING OF DIGITAL THERAPEUTICS}

Digital therapeutics (DTx) one of the most recent frontiers of medicine and can be defined as "technologies that deliver medical interventions directly to patients using evidence-based, clinically evaluated software to treat, manage, and prevent a broad spectrum of diseases and disorders" (Digital Therapeutics Alliance, 2021).

As for conventional medicines, with the increasing uptake of DTx into clinical practice, a proper post-marketing surveillance of DTx has to be implemented to rapidly identify potential safety signals and establish the safety profile of these technologies. Side effects associated with DTx may be generally less severe and easier to manage than those caused by conventional drugs. However, based on findings from pivotal trials, adverse effects of DTx may still occur to a greater extent than in respective control arms, thus requiring careful post-marketing monitoring.

Another important aspect of DTx is that they allow to collect a massive quantity of post-marketing patient-level data that can be harnessed to re-assess their safety and effectiveness in real-world setting. However, the increase in individual patient-related data poses concerns about data privacy and quality, thus highlighting the need to define a legal framework that allows on the one hand to guarantee individual privacy and on the other hand to transparently share data for research purposes.

\section{PHARMACOVIGILANCE OF ADVANCED THERAPY MEDICINAL PRODUCTS}

Advanced therapy medicinal products (ATMPs) are medicines for human use that are based on genes, cells or tissue engineering (European Medicines Agency, 2021). ATMPs provide new opportunities to restore, correct or modify physiological functions or make a medical diagnosis. Due to their high innovativeness, these medicines usually benefit from accelerated assessment and accelerated approval pathways, thus highlighting the need to generate post-marketing evidence about their benefit-risk profile. However, uncertainties concerning the safety profile of new ATMPs cannot be ascribed only to regulatory pathways. As these medicines often target rare diseases, pre-marketing evidence is generally weak because of inherent limitations of clinical trials due to small number of recruited patients, use of surrogate endpoints and single-arm design (Augustine et al., 2013). Therefore, post-marketing studies play a key role in generating long term evidence about the safety of these medicines and to fill the knowledge gap of pre-marketing studies. The detection of safety issues should start early and continue throughout the development of the ATMP in order to prevent or minimize the risk when possible. In some cases, the use of ATMPs is expected to be a once in a life-time treatment, therefore the sustainability of efficacy over time is a question that can only be answered by long-term efficacy follow-up. The objectives of the safety and efficacy follow-up will depend on the characteristics of the product (European Medicines Agency, 2018a). In the case of chimeric antigenic therapies (CAR-T) routine risk minimization measures have to be supplemented with additional risk minimization measures under relevant important risks (e.g., cytokine release syndrome, infections and serious neurological adverse reactions) (European Medicines Agency, 2018b; European Medicines Agency, 2018c).

\section{ECOPHARMACOVIGILANCE}

Ecopharmacovigilance is "the science and activities concerning detection, assessment, understanding and prevention of adverse effects or other problems related to the presence of pharmaceuticals in the environment, which affect both human and the other animal species" (Velo and Moretti, 2010), ecopharmacovigilance is a very important issue nowadays and it plays a crucial role to reduce the environmental risk of pharmaceutical pollutants. Indeed, pharmaceuticals are widespread environmental pollutants that may be excreted into the environment through different routes, such as the excretion by the patient as parent compound or active metabolites via the sewer system and the release into the waste waters by manufacturers or hospitals and the terrestrial depositions (Holm et al., 2013). Several studies documented the effects of pharmaceutical pollution on various animal species, such as vultures and fish (Wang et al., 2017). The role of ecopharmacovigilance is becoming more and more important to control and minimize the sources of pharmaceutical pollution through the detection, assessment and prevention of adverse effects related to the presence of pharmaceuticals in the environment.

Although the detected concentrations of pharmaceuticals in the environment were mainly low (ng/L to $\mu \mathrm{g} / \mathrm{L}$ ) potential direct and indirect risks for humans exist and should be carefully monitored. Indeed, it is known that sex hormones exert their pharmacological activity at very low concentrations and that exposure to antibiotics may contribute to bacterial resistance (Velo and Moretti, 2010). Furthermore, special populations like pregnant women, children and older patients may be more vulnerable also to low concentrations of medicines. Addressing issues related to pharmaceutical pollution is therefore one of the main current aims of pharmacovigilance.

At Frontiers in Drug Safety and Regulation, we are interested in promoting research concerning the disciplines of pharmacovigilance, pharmacoepidemiology, regulatory science and public health to elevate regulatory sciences with patientoriented approaches addressing emerging issues concerning drug safety.

\section{AUTHOR CONTRIBUTIONS}

GT and SC conceived the study and wrote the paper. 


\section{REFERENCES}

Augustine, E. F., Adams, H. R., and Mink, J. W. (2013). Clinical Trials in Rare Disease: Challenges and Opportunities. J. Child. Neurol. 28 (9), 1142-1150. doi:10.1177/0883073813495959

Basile, A. O., Yahi, A., and Tatonetti, N. P. (2019). Artificial Intelligence for Drug Toxicity and Safety. Trends Pharmacol. Sci. 40 (9), 624-635. doi:10.1016/j.tips. 2019.07.005

Bate, A., and Hobbiger, S. F. (2021). Artificial Intelligence, Real-World Automation and the Safety of Medicines. Drug Saf. 44 (2), 125-132. doi:10.1007/s40264020-01001-7

Crisafulli, S., Ientile, V., L’Abbate, L., Fontana, A., Linguiti, C., Manna, S., et al. (2021). COVID-19 Patient Management in Outpatient Setting: A PopulationBased Study from Southern Italy. Jcm 11 (1), 51. doi:10.3390/jcm11010051

Digital Therapeutics Alliance (2021). Understanding DTx: A New Category of Medicine. Available at: https://dtxalliance.org/understanding-dtx/. (Accessed January 30, 2022).

European Medicines Agency (2021). Advanced Therapy Medicinal Products: Overview. Available at: https://www.ema.europa.eu/en/human-regulatory/ overview/advanced-therapy-medicinal-products-overview.

European Medicines Agency (2018a). Guideline on Safety and Efficacy Follow-Up and Risk Management of Advanced Therapy Medicinal Products. Available at: https://www.ema.europa.eu/en/documents/scientific-guideline/draft-guidelinesafety-efficacy-follow-risk-management-advanced-therapy-medicinal-productsrevision_en.pdf (Accessed January 30, 2022).

European Medicines Agency (2018c). Summary of Risk Management Plan for Yescarta (Axicabtagene Ciloleucel). Available at: https://www.ema.europa.eu/ en/documents/rmp-summary/yescarta-epar-risk-management-plan-summary_ en.pdf. (Accessed January 30, 2022).

European Medicines Agency (2018b). Summary of the Risk Management Plan for Kymriah (Tisagenlecleucel). Available at: https://www.ema.europa.eu/en/ documents/rmp-summary/kymriah-epar-risk-management-plan-summary en.pdf. (Accessed January 30, 2022).

Food and Drug Administration (2019). FDA's Sentinel Initiative. Available at: https:// www.fda.gov/safety/fdas-sentinel-initiative. (Accessed January 30, 2022).

Gini, R., Sturkenboom, M. C. J., Sultana, J., Cave, A., Landi, A., Pacurariu, A., et al. (2020). Different Strategies to Execute Multi-Database Studies for Medicines Surveillance in Real-World Setting: A Reflection on the European Model. Clin. Pharmacol. Ther. 108 (2), 228-235. doi:10.1002/cpt.1833

Holm, G., Snape, J. R., Murray-Smith, R., Talbot, J., Taylor, D., and Sörme, P. (2013). Implementing Ecopharmacovigilance in Practice: Challenges and Potential Opportunities. Drug Saf. 36 (7), 533-546. doi:10.1007/s40264-013-0049-3

Lane, J. C. E., Weaver, J., Kostka, K., Duarte-Salles, T., Abrahao, M. T. F., Alghoul, H., et al. (2020). Risk of Hydroxychloroquine Alone and in Combination with Azithromycin in the Treatment of Rheumatoid Arthritis: a Multinational, Retrospective studyThe Lancet. Lancet Rheumatol. 2 (11), e698-e711. doi:10. 1016/S2665-9913(20)30276-9

Liu, M., Caputi, T. L., Dredze, M., Kesselheim, A. S., and Ayers, J. W. (2020). Internet Searches for Unproven COVID-19 Therapies in the United States. JAMA Intern. Med. 180 (8), 1116-1118. doi:10.1001/jamainternmed.2020.1764

Niburski, K., and Niburski, O. (2020). Impact of Trump's Promotion of Unproven COVID-19 Treatments on Social Media and Subsequent Internet Trends: Observational Study. J. Med. Internet Res. 22 (11), e20044. doi:10.2196/20044

Singh, S., and Loke, Y. K. (2012). Drug Safety Assessment in Clinical Trials: Methodological Challenges and Opportunities. Trials 13, 138. doi:10.1186/ 1745-6215-13-138
Spila Alegiani, S., Crisafulli, S., Giorgi Rossi, P., Mancuso, P., Salvarani, C., Atzeni, F., et al. (2021). Risk of Coronavirus Disease 2019 Hospitalization and Mortality in Rheumatic Patients Treated with Hydroxychloroquine or Other Conventional Disease-Modifying Anti-rheumatic Drugs in Italy. Rheumatology 60 (SI), SI25-SI36. doi:10.1093/rheumatology/keab348

Sultana, J., Crisafulli, S., Gabbay, F., Lynn, E., Shakir, S., and Trifirò, G. (2020a). Challenges for Drug Repurposing in the COVID-19 Pandemic Era. Front. Pharmacol. 11, 588654. doi:10.3389/fphar.2020.588654

Sultana, J., Cutroneo, P. M., Crisafulli, S., Puglisi, G., Caramori, G., and Trifirò, G. (2020b). Azithromycin in COVID-19 Patients: Pharmacological Mechanism, Clinical Evidence and Prescribing Guidelines. Drug Saf. 43 (8), 691-698. doi:10. 1007/s40264-020-00976-7

Trifirò, G., Isgrò, V., Ingrasciotta, Y., Ientile, V., L'Abbate, L., Foti, S. S., et al. (2021). Large-Scale Postmarketing Surveillance of Biological Drugs for Immune-Mediated Inflammatory Diseases through an Italian Distributed Multi-Database Healthcare Network: The VALORE Project. BioDrugs 35 (6), 749-764. doi:10.1007/s40259-021-00498-3

Trifirò, G., Massari, M., Da Cas, R., Menniti Ippolito, F., Sultana, J., Crisafulli, S., et al. (2020). Renin-Angiotensin-Aldosterone System Inhibitors and Risk of Death in Patients Hospitalised with COVID-19: A Retrospective Italian Cohort Study of 43,000 Patients. Drug Saf. 43 (12), 1297-1308. doi:10.1007/s40264020-00994-5

Velo, G., and Moretti, U. (2010). Ecopharmacovigilance for Better Health. Drug Saf. 33 (11), 963-968. doi:10.2165/11539380-000000000-00000

Wang, J., He, B., Yan, D., and Hu, X. (2017). Implementing Ecopharmacovigilance (EPV) from a Pharmacy Perspective: A Focus on Non-steroidal Antiinflammatory Drugs. Sci. Total Environ. 603-604, 772-784. doi:10.1016/j. scitotenv.2017.02.209

Williamson, E. J., Walker, A. J., Bhaskaran, K., Bacon, S., Bates, C., Morton, C. E., et al. (2020). Factors Associated with COVID-19-Related Death Using OpenSAFELY. Nature 584 (7821), 430-436. doi:10.1038/s41586-0202521-4

Wong, A., Plasek, J. M., Montecalvo, S. P., and Zhou, L. (2018). Natural Language Processing and its Implications for the Future of Medication Safety: A Narrative Review of Recent Advances and Challenges. Pharmacotherapy 38 (8), 822-841. doi:10.1002/phar.2151

World Health Organization (2020). Risk Communication and Community Engagement Readiness and Response to Coronavirus Disease (COVID-19). Available at: file:///C:/Users/Farmacologia/Downloads/WHO-2019-nCoVRCCE-2020.2-eng.pdf. (Accessed January 30, 2022).

Conflict of Interest: The authors declare that the research was conducted in the absence of any commercial or financial relationships that could be construed as a potential conflict of interest.

Publisher's Note: All claims expressed in this article are solely those of the authors and do not necessarily represent those of their affiliated organizations, or those of the publisher, the editors and the reviewers. Any product that may be evaluated in this article, or claim that may be made by its manufacturer, is not guaranteed or endorsed by the publisher.

Copyright $\odot 2022$ Trifirò and Crisafulli. This is an open-access article distributed under the terms of the Creative Commons Attribution License (CC BY). The use, distribution or reproduction in other forums is permitted, provided the original author(s) and the copyright owner(s) are credited and that the original publication in this journal is cited, in accordance with accepted academic practice. No use, distribution or reproduction is permitted which does not comply with these terms. 